\title{
Comparison of high-order curved finite elements
}

\author{
Ruben Sevilla*, ${ }^{\dagger}$, Sonia Fernández-Méndez and Antonio Huerta \\ Laboratori de Càlcul Numèric (LaCàN), Departament de Matemàtica Aplicada III, E.T.S. de Ingenieros de \\ Caminos, Canales y Puertos, Universitat Politècnica de Catalunya, Jordi Girona 1, E-08034 Barcelona, Spain
}

\begin{abstract}
SUMMARY
Several finite element techniques used in domains with curved boundaries are discussed and compared, with particular emphasis in two issues: the exact boundary representation of the domain and the consistency of the approximation. The influence of the number of integration points on the accuracy of the computation is also studied. Two-dimensional numerical examples, solved with continuous and discontinuous Galerkin formulations, are used to test and compare all these methodologies. In every example shown, the recently proposed NURBS-enhanced finite element method (NEFEM) provides the maximum accuracy for a given spatial discretization, at least one order of magnitude more accurate than classical isoparametric finite element method (FEM). Moreover, NEFEM outperforms Cartesian FEM and $p$-FEM, stressing the importance of the geometrical model as well as the relevance of a consistent approximation in finite element simulations. Copyright (C) 2011 John Wiley \& Sons, Ltd.
\end{abstract}

Received 17 February 2010; Revised 14 December 2010; Accepted 14 December 2010

KEY WORDS: finite element method; isoparametric FEM; Cartesian FEM; $p$-version FEM; NURBSenhanced FEM; exact geometry

\section{INTRODUCTION}

An accurate geometric description of a domain with curved boundaries is critical in the so-called $p$ extensions of the finite element method (FEM), see [1]. In this approach the mesh remains unchanged (usually containing elements with a large aspect ratio) and the polynomial order of the approximation is increased in order to properly approximate the solution. In some applications, geometric errors induced by the isoparametric mapping deteriorate the accuracy of the numerical solution, see [1-3]. Therefore, an accurate description of the geometry is mandatory in order to obtain maximum accuracy for a given spatial discretization. Thus, in the so-called $p$-version of FEM ( $p$-FEM, see [4]) the blending function method [5] is usually considered to define an exact mapping relating local and Cartesian coordinates. This mapping offers advantages compared to classical isoparametric mapping, see for instance [2].

Non-uniform rational B-splines (NURBS, see [6]) are widely used for geometry description in computer-aided design (CAD). Other popular options for geometric description in CAD are polynomial B-splines (a particular case of NURBS) and subdivision surfaces, see [7]. This fact has motivated novel numerical methodologies considering exact CAD descriptions of the computational domain. Numerical methods using an exact geometric model can be classified into two categories.

The first category considers methods with an exact CAD description of the entire computational domain and approximation functions defined with the same basis used for the CAD representation

\footnotetext{
${ }^{*}$ Correspondence to: Ruben Sevilla, Laboratori de Càlcul Numèric (LaCàN), E.T.S. Ingenieros de Caminos, Universitat Politècnica de Catalunya, Jordi Girona 1, E-08034 Barcelona, Spain.

$\dagger$ E-mail: ruben.sevilla@upc.edu
} 
of the geometry, instead of the classical piecewise polynomial approximation. They are referred as isogeometric methods. For instance, in [8] polynomial B-splines are used both to describe the geometric model and to approximate the solution. In [9] the same idea is implemented with subdivision surfaces, and further applied to thin shell analysis. More recently, the method proposed in [10] and the isogeometric analysis [11] follow the same rationale, considering NURBS for the geometric description as well as for the analysis.

The second category also considers an exact geometric model. The boundaries of the computational domain are exactly represented and a standard finite element (FE) rationale is used. That is, a standard piecewise polynomial approximation of the solution is considered. First attempts to combine NURBS and $p$-FEM can be found in the context of shape optimization, see [12] and references therein. The recently proposed NURBS-enhanced finite element method (NEFEM), see $[3,13]$, also lies in this category. NEFEM uses NURBS to exactly describe the boundary of the computational domain. In a sense, it is similar to $p$-FEM using NURBS to exactly capture the boundary. More precisely, the main difference between NEFEM and $p$-FEM is that NEFEM approximates the solution directly with Cartesian coordinates (not in a reference element), ensuring reproducibility of polynomials in the physical space. Moreover, from a practical point of view, efficient strategies are proposed in NEFEM for the numerical integration on elements affected by the NURBS boundary representation.

This paper is restricted to methods falling in the second category. Such methods are simpler and more efficient because of two crucial aspects. First, NURBS functions are only used to describe the geometry of curved boundaries, which is the geometry description provided by commercial CAD software, whereas isogeometric methods require a NURBS solid for three-dimensional (3D) simulations. Second, methods such as NEFEM or p-FEM use standard FE polynomial interpolation

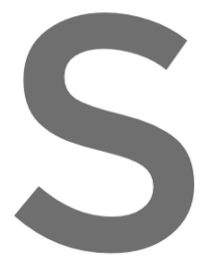
and numerical integration. Thus, in the large majority of the domain (namely in the intertor, that is, for elements not in computational efficien NURBS are used functions over NURBS or $p$-FEM, which requ intersecting
ncy of the appro
s domains
uires integr s, in the large maj
classical FEM. The curvel bound
oximating the solut
s, being more expenting polynomial ajority of the dom
hdary) standard F
his is not the case
ution. Those metl
pensive than the 1
l functions over do are considered, prese
in isogeometric analys
ods require integrating
umerical integration in
mains with NURBS bo

Moreover, it is worth mentioning that the tensor product nature of isogeometric methods allow an

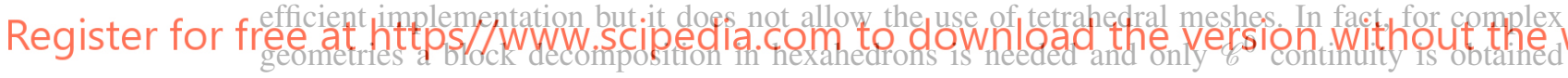
watermark between blocks, whereas with NEFEM standard tetrahedral meshes can be considered. Finally, isogeometric analysis requires a specific treatment of trimmed NURBS surfaces, see [14], whereas trimmed NURBS are easily handled in the NEFEM context, see [15].

Several FE methodologies for the treatment of curved boundaries are considered and compared; namely, classical isoparametric FEM and Cartesian FEM with an approximated boundary representation, and $p$-FEM and NEFEM with an exact description of boundary. In Section 2 the basis of each methodology is recalled. Special attention is paid to numerical integration for methods that consider an exact description of the geometry with NURBS. A critical comparison between these methods is also presented. Section 3 presents two numerical examples in two dimensions, which are solved with continuous and discontinuous Galerkin (DG) formulations. The performance and benefits of each FE methodology are compared and discussed.

\section{FE METHODS IN DOMAINS WITH CURVED BOUNDARIES}

Let $\Omega \subset \mathbb{R}^{2}$ be an open bounded domain whose boundary $\partial \Omega$, or a portion of it, is curved. A regular partition of the domain $\bar{\Omega}=\bigcup_{e} \bar{\Omega}_{e}$ in subdomains, triangles in this work, is assumed, such that $\Omega_{i} \cap \Omega_{j}=\emptyset$, for $i \neq j$. For instance, Figure 1 shows a domain with part of the boundary described by a NURBS curve corresponding to a circle, and a triangulation of the domain with curved FEs. It is important to remark that, in the following, $\Omega_{e}$ denotes the element with an exact description of the curved boundary, also referred as physical subdomain. This is not the case of 

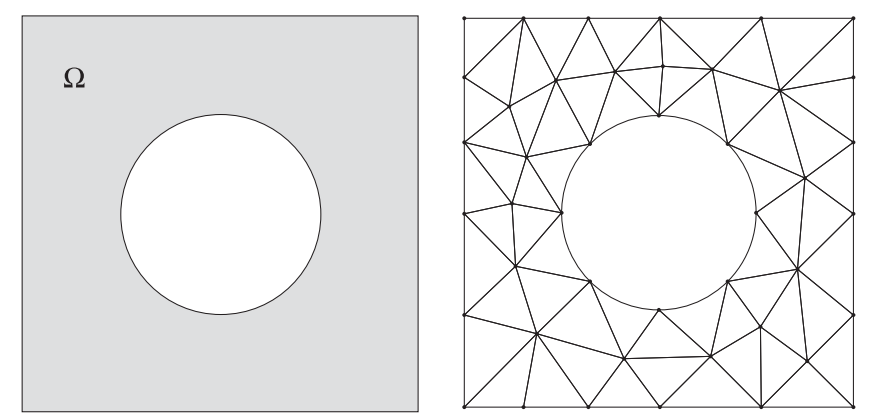

Figure 1. Physical domain $\Omega$ with a curved boundary (left) and a triangulation of the domain with curved FEs (right).

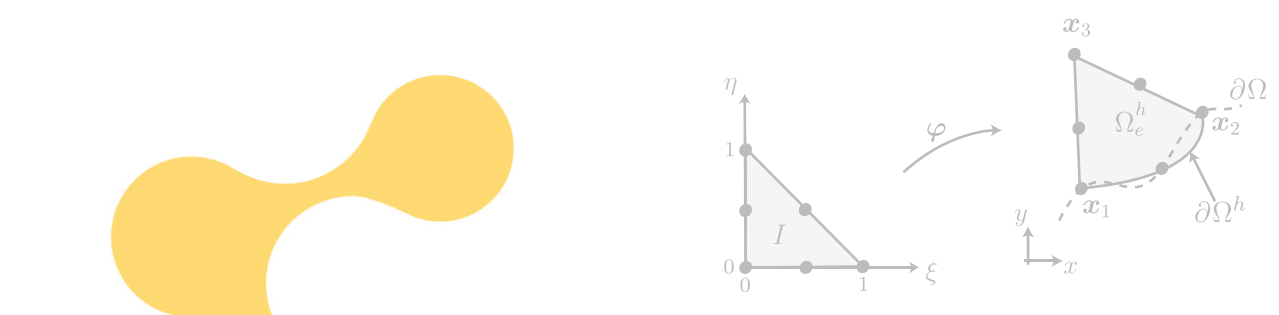

Figure 2. Isoparametric mapping between the reference element $I$, with local coordinates $\xi$, and an
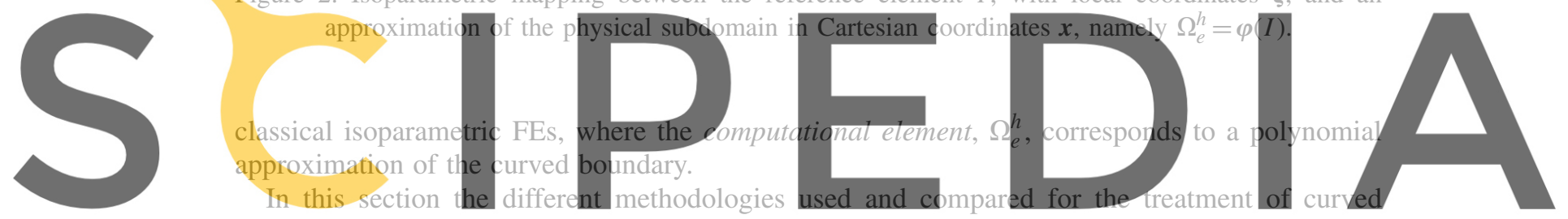

boundaries are recalled.

Register for free at https//www.scipedia.com to download the version without the watermark

2.1. Approximated boundary representation

The standard FE technique used in the presence of curved boundaries is the isoparametric FEM, see $[16,17]$. A nodal interpolation of the solution, $u$, is considered in the reference element $I$ with local coordinates $\xi=(\xi, \eta)$, see Figure 2,

$$
u(\xi) \simeq u^{h}(\xi)=\sum_{i=1}^{\mathrm{n}_{\mathrm{en}}} u_{i} N_{i}(\xi)
$$

where $u_{i}$ are nodal values, $N_{i}$ are polynomial shape functions of order $p$ in $\xi$, and nen is the number of element nodes. The isoparametric transformation is used to relate local and Cartesian coordinates

$$
\begin{aligned}
\varphi: I \longrightarrow \Omega_{e}^{h} \\
\xi \\
\quad \longmapsto \varphi(\xi):=\sum_{i=1}^{\mathrm{n}_{\mathrm{en}}} \boldsymbol{x}_{i} N_{i}(\xi),
\end{aligned}
$$

where $\boldsymbol{x}_{i}$ are the nodal coordinates of the computational element $\Omega_{e}^{h}$. Note that $\Omega_{e}^{h}$ is a polynomial approximation of the physical subdomain $\Omega_{e}$, in particular, of its boundary, see Figure 2 . In fact, the term isoparametric stands for the use of the same polynomial shape functions to define the functional approximation $u^{h}$, and to describe the geometry of the computational element in Cartesian coordinates, see Equation (2). 
Numerical integration in the computational element $\Omega_{e}^{h}$ (approximation of $\Omega_{e}$ ) is performed using the isoparametric transformation given in Equation (2), with a numerical quadrature in the reference element $I$. For instance, a stiffness elemental matrix coefficient is computed as

$$
K_{i j}^{e}=\int_{\Omega_{e}^{h}} \nabla_{\boldsymbol{x}} N_{i}(\boldsymbol{\xi}(\boldsymbol{x})) \cdot \nabla_{\boldsymbol{x}} N_{i}(\boldsymbol{\xi}(\boldsymbol{x})) \mathrm{d} \Omega=\int_{I}\left(\boldsymbol{J}_{\boldsymbol{\varphi}}^{-1} \nabla_{\boldsymbol{\xi}} N_{i}(\boldsymbol{\xi})\right) \cdot\left(\boldsymbol{J}_{\boldsymbol{\varphi}}^{-1} \nabla_{\boldsymbol{\xi}} N_{j}(\boldsymbol{\xi})\right)\left|\boldsymbol{J}_{\boldsymbol{\varphi}}\right| \mathrm{d} \boldsymbol{\xi},
$$

where $\boldsymbol{J}_{\boldsymbol{\varphi}}$ is the Jacobian of the isoparametric transformation, see Equation (2). For curved elements the isoparametric mapping is non-linear. Therefore, the inverse of the Jacobian, $\boldsymbol{J}_{\boldsymbol{\varphi}}^{-1}$, is not a polynomial function, and no exact integration is feasible with standard quadrature rules. In practice, a symmetric triangle quadrature [18] on $I$, with a sufficiently large number of integration points, is usually employed to compute (3). In fact, a quadrature of order $2 p-1$ provides optimal convergence of the isoparametric FEM, see [19].

There are two sources of error in isoparametric FEM. First, the isoparametric mapping in Equation (2) introduces geometric errors, due to the approximation of the physical subdomain $\Omega_{e}$ by the computational element $\Omega_{e}^{h}$. In fact, the boundary of the computational domain $\partial \Omega_{h}$ is a piecewise polynomial approximation of the exact boundary $\partial \Omega$, see Figure 2. Second, for high-order approximations on curved elements, the definition of the polynomial interpolation in Equation (1) in local coordinates, $\boldsymbol{\xi}$, implies a loss of consistency: a polynomial interpolation of degree $p>1$ in $\xi$ does not correspond to a polynomial interpolation of degree $p$ in $x$. This implies that the approximation is able to reproduce linear functions but it is not able to reproduce higher order polynomials in Cartesian coordinates. In other words, curved isoparametric FEs pass the patch test but they fail to pass the so-called higher order patch tests, see [19] for further details.
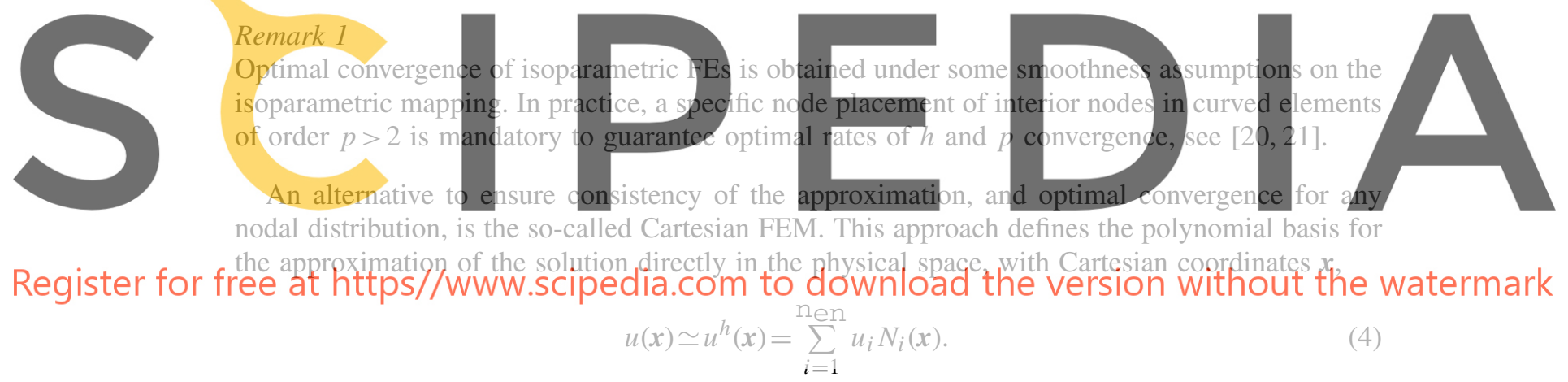

Nevertheless, the isoparametric transformation in Equation (2) and the computational element $\Omega_{e}^{h}$ are still considered for integration purposes. For instance, a stiffness elemental matrix coefficient is computed as

$$
K_{i j}^{e}=\int_{\Omega_{e}^{h}} \nabla_{\boldsymbol{x}} N_{i}(\boldsymbol{x}) \cdot \nabla_{\boldsymbol{x}} N_{j}(\boldsymbol{x}) d \Omega=\int_{I} \nabla_{\boldsymbol{x}} N_{i}(\boldsymbol{x}(\boldsymbol{\xi})) \cdot \nabla_{\boldsymbol{x}} N_{j}(\boldsymbol{x}(\boldsymbol{\xi}))\left|\boldsymbol{J}_{\boldsymbol{\varphi}}\right| \mathrm{d} \boldsymbol{\xi} .
$$

The definition of the approximation with Cartesian coordinates, $\boldsymbol{x}$, ensures reproducibility of polynomials (i.e. consistency of order $p$ ). Moreover, exact integration is feasible because shape functions and their derivatives are polynomials, not only with Cartesian coordinates $\boldsymbol{x}$, but also with local coordinates $\xi$. More precisely, for a degree of interpolation $p, N_{i}(\boldsymbol{x}(\xi))$ and $\nabla_{\boldsymbol{x}} N_{i}(\boldsymbol{x}(\boldsymbol{\xi}))$ are polynomials of degree $p$ and $p-1$, respectively. Therefore, the function to be integrated, $f(\boldsymbol{\xi})=\nabla_{\boldsymbol{x}} N_{i}(\boldsymbol{x}(\boldsymbol{\xi})) \cdot \nabla_{\boldsymbol{x}} N_{j}(\boldsymbol{x}(\boldsymbol{\xi}))\left|\boldsymbol{J}_{\boldsymbol{\varphi}}\right|$, is a polynomial of degree $p(4 p-3)$ in $\boldsymbol{\xi}$. The evaluation of integral in Equation (5) can be exact with a triangle quadrature of order $p(4 p-3)$ on the reference element $I$.

It is worth noting that Cartesian approximation can be considered with Lagrangian, Eulerian, arbitrary Lagrangian-Eulerian, or updated Lagrangian formulations. Obviously, Cartesian approximation introduces an overhead with respect to isoparametric FEs because it requires a specific definition of the approximation for each curved element. When the mesh is fixed, this overhead is restricted to elements affected by the curved boundary description, usually a very small portion of 
the total number of elements. For meshes evolving with the simulation, this overhead is repeated each time step, and internal curved edges must be considered. The definition of Cartesian approximations for elements with internal curved edges does not represent an excessive difficulty and it follows the ideas presented in the extension of NEFEM to 3D domains [22], where internal curved faces have to be considered. It is worth remarking that the extra cost introduced by Cartesian FEM is justified by the improved accuracy with respect to isoparametric FEs. In addition, Cartesian approximation allows to ensure optimal convergence with no dependence on the node placement for curved elements, see Remark 1 and Section 2.4.

Although Cartesian FEM ensures reproducibility of polynomials in the physical space, the numerical integration in Cartesian FEM is still done in the (approximated) computational element $\Omega_{e}^{h}$. Thus, Cartesian FEM precludes the lack consistency of isoparametric FEM, but it still suffers from geometric error. This is not the case for $p$-FEM and NEFEM formulations, with the exact boundary representation, described next.

\subsection{Exact boundary representation}

This section recalls the basics of two formulations considering an exact boundary representation, $p$-FEM [1,4] and NEFEM [3]. In order to simplify the presentation and without loss of generality, curved physical subdomains, $\Omega_{e}$, are assumed to be triangles with one curved edge.

Nodal interpolation in $p$-FEM is defined in the reference element using local coordinates $\xi$, see Equation (1), but an exact mapping is employed between the reference element $I$ and the physical subdomain $\Omega_{e}$. For instance, assuming a NURBS parametrization $C(\xi)$ of the curved edge of $\Omega_{e}$, a simple $p$-FEM mapping may be
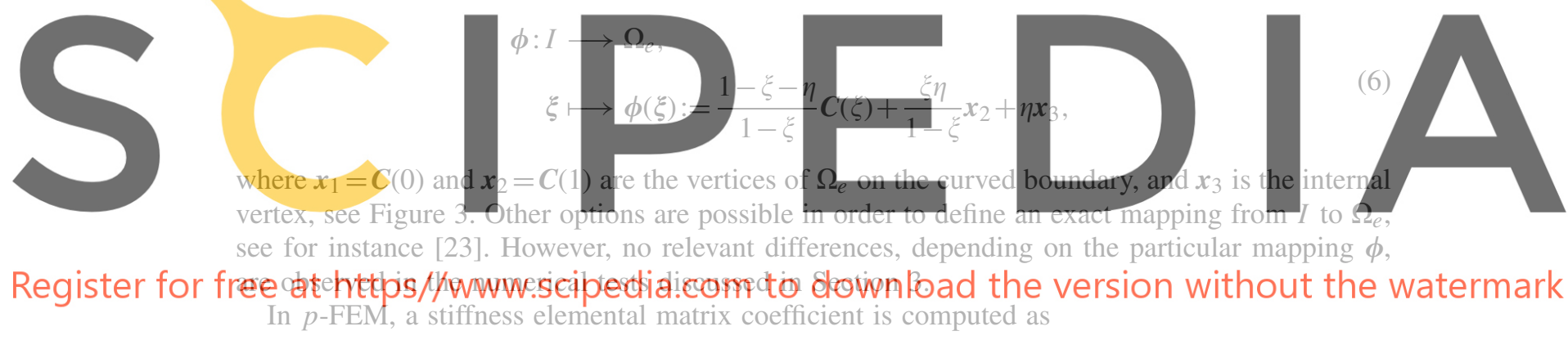

$$
K_{i j}^{e}=\int_{\Omega_{e}} \nabla_{\boldsymbol{x}} N_{i}(\boldsymbol{\xi}(\boldsymbol{x})) \cdot \nabla_{\boldsymbol{x}} N_{i}(\boldsymbol{\xi}(\boldsymbol{x})) \mathrm{d} \Omega=\int_{I}\left(\boldsymbol{J}_{\boldsymbol{\phi}}^{-1} \nabla_{\boldsymbol{\xi}} N_{i}(\boldsymbol{\xi})\right) \cdot\left(\boldsymbol{J}_{\boldsymbol{\phi}}^{-1} \nabla_{\boldsymbol{\xi}} N_{j}(\boldsymbol{\xi})\right)\left|\boldsymbol{J}_{\boldsymbol{\phi}}\right| \mathrm{d} \boldsymbol{\xi},
$$

integrating over the physical subdomain $\Omega_{e}$, with an exact description of the geometry. Note that, the inverse of the Jacobian, $\boldsymbol{J}_{\phi}^{-1}$ is not a polynomial function and, as for the isoparametric FEM, no exact integration is feasible with standard quadrature rules. Nevertheless, under some smoothness requirements on the parametrization $\boldsymbol{C}(\xi)$, the same quadrature order used in the isoparametric FEM, that is $2 p-1$, guarantees optimal convergence for $p$-FEM, see [24].

Note that $p$-FEM presents a major advantage, compared to isoparametric or Cartesian FEM, which is the exact boundary representation. Nevertheless, $p$-FEM still suffers the same lack of

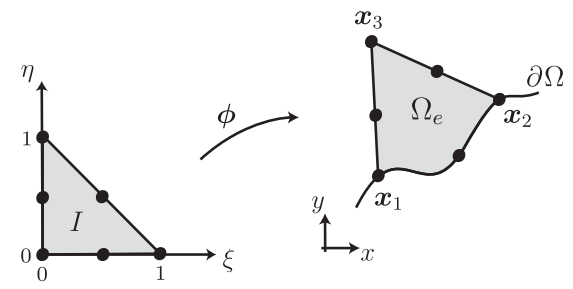

Figure 3. Exact mapping between the reference element $I$ with local coordinates $\xi$, and the physical subdomain $\Omega_{e}$ with Cartesian coordinates $\boldsymbol{x}$. 


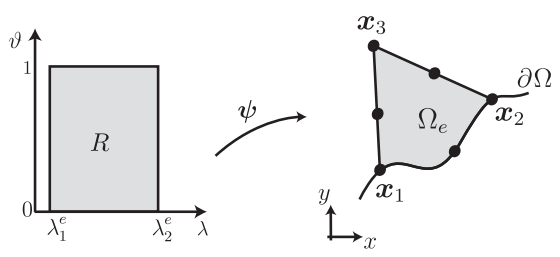

Figure 4. Transformation from $R=\left[\lambda_{1}^{e}, \lambda_{2}^{e}\right] \times[0,1]$ to $\Omega_{e}$ for numerical integration in the 2D NEFEM.

consistency as isoparametric FEM, due to the definition of the polynomial shape functions in the reference element $I$, with local coordinates $\xi$. This is not the case for the recently proposed NEFEM [3].

NEFEM considers the exact geometry description by means of the usual CAD boundary representation of the computational domain with NURBS. The polynomial approximation in Equation (4) is defined with Cartesian coordinates $\boldsymbol{x}$, ensuring reproducibility of polynomials in the physical space for any order of approximation $p$. The exact description of the boundary is used to perform the numerical integration on the physical subdomain $\Omega_{e}$. In NEFEM, a stiffness elemental matrix coefficient is computed as

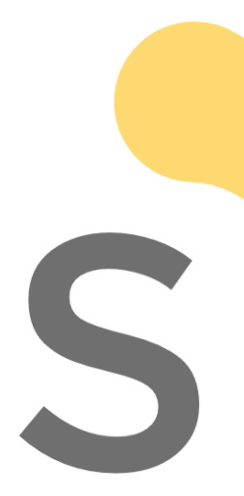

$$
K_{i j}^{e}=\int_{\Omega_{e}} \nabla_{x} N_{i}(\boldsymbol{x}) \cdot \nabla_{x} N_{j}(\boldsymbol{x}) \mathrm{d} \Omega=\int_{R} \nabla_{x} N_{i}(\boldsymbol{x}(\boldsymbol{\lambda})) \cdot \nabla_{x} N_{j}(\boldsymbol{x}(\boldsymbol{\lambda}))\left|\boldsymbol{J}_{\psi}\right| \mathrm{d} \lambda,
$$

using the transformation

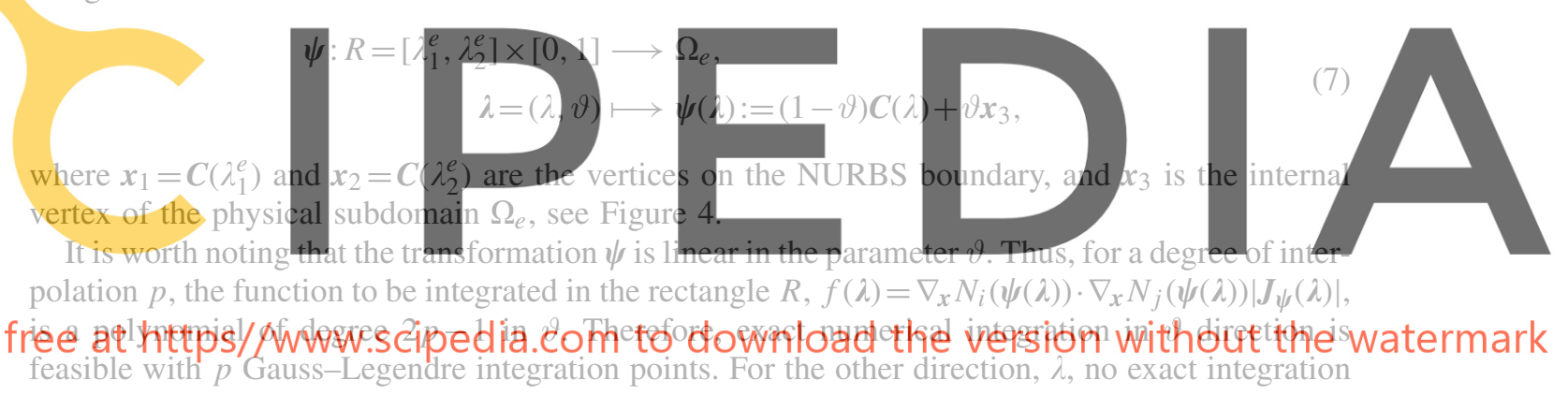

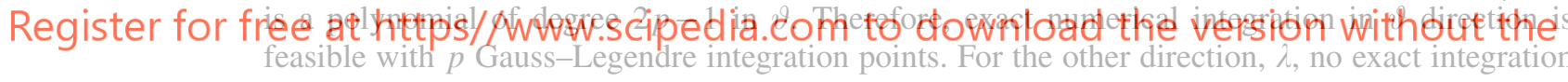
is feasible with standard quadratures due to the rational definition of the NURBS boundary. Numerical experiments reveal that Gauss-Legendre quadratures are a competitive choice in front of other quadrature rules such as trapezoidal and Simpson composite rules or Romberg's integration, see [3]. Numerical examples in Section 3.1 show the influence of the number of integration points on the accuracy of NEFEM computations.

It is worth mentioning that NURBS are piecewise rational functions defined in parametric form, see [6]. Therefore, numerical integration for $p$-FEM and NEFEM must be designed to account for changes of NURBS definition along the curved edge of the physical subdomain $\Omega_{e}$. This issue is addressed in the next section.

\subsection{Numerical integration for $p$-FEM and NEFEM}

This section discusses the numerical integration for $p$-FEM and NEFEM when changes of NURBS parametrization are considered inside the curved boundary edge of a physical subdomain $\Omega_{e}$ in 2D. Key ideas for an extension to 3D domains are also discussed.

For illustration purposes the triangle with a curved edge represented in Figure 5 is considered first. The curved edge is described with a piecewise parametrization $\boldsymbol{C}$, whose definition changes in two points on the curved edge, marked with $\square$. The parametric coordinates of these points are called the breakpoints or knots of the NURBS parametrization, see [6].

In $p$-FEM, the piecewise definition of the boundary induces a piecewise definition of the mapping $\phi$, see Equation (6). Therefore, a specifically designed numerical quadrature should be defined in the reference element $I$. For the triangle represented in Figure 5, with two changes of 


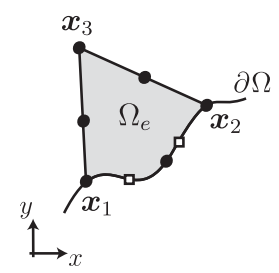

Figure 5. Triangle with a curved edge containing changes of NURBS definition (marked with $\square$ ).

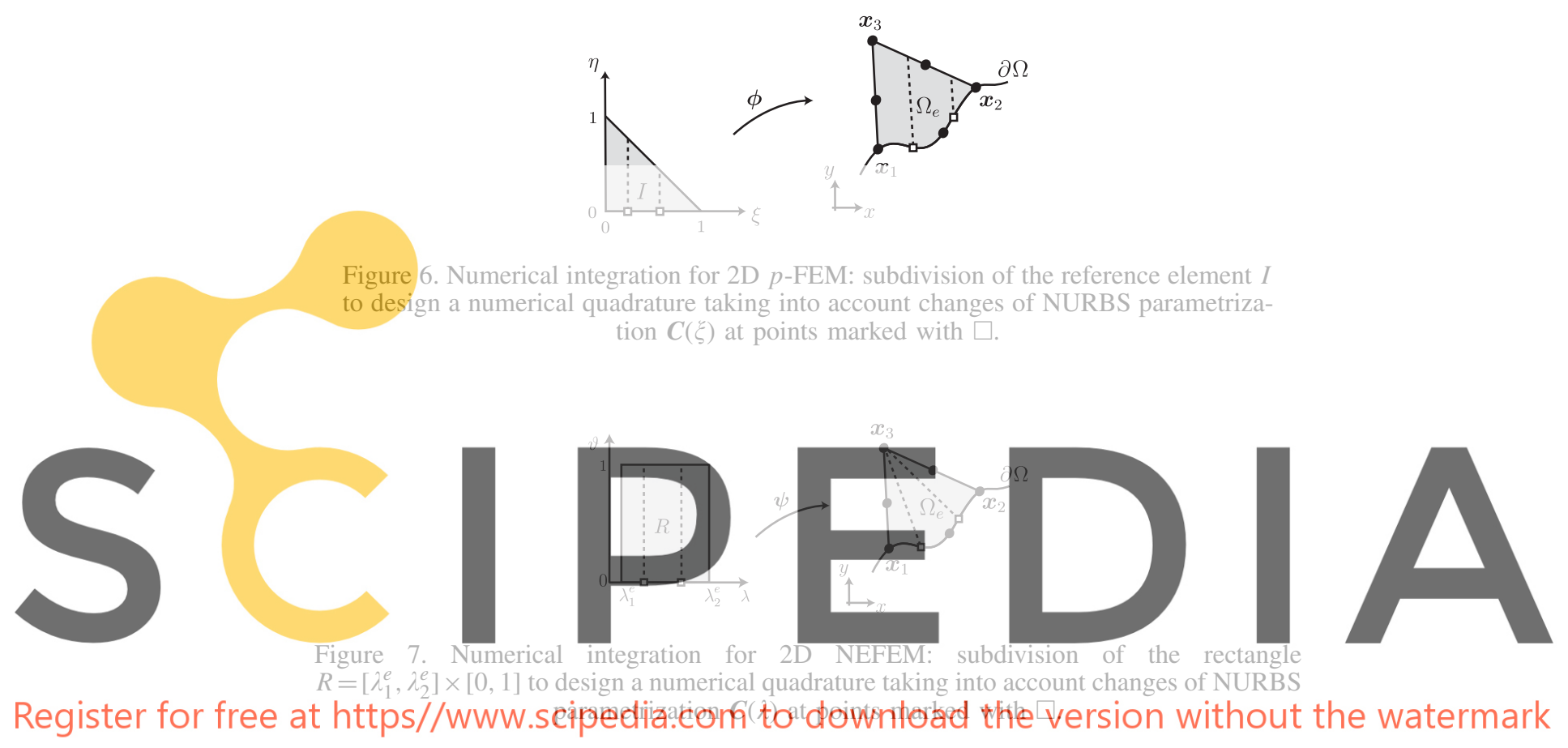

NURBS definition, the reference element should be partitioned as represented in Figure 6, where the discontinuous lines show the changes of definition of the mapping $\phi$. Note that these lines originate at the breakpoints of the NURBS parametrization in the $\xi$ axis, and are extended inside the reference element. A composite numerical quadrature on $I$ should be defined by using different numerical quadratures in each region.

In NEFEM, changes of NURBS definition are easily accommodated using application $\psi$, see Equation (7). The piecewise definition of the boundary also induces a piecewise definition of the element mapping $\psi$. The rectangle $R$ is subdivided using the breakpoints, as represented in Figure 7 , and a numerical quadrature in $R$ is defined only in terms of 1D quadratures. A composite 1D Gauss-Legendre quadrature is used in parameter $\lambda$ to take into account the discontinuous nature of the NURBS parametrization. In the other parameter, $\vartheta$, exact integration is feasible, as discussed in Section 2.2.

The proposed strategy to perform the numerical integration in 2D NEFEM can be easily extended to 3D domains, see [22]. For instance, let $\Omega_{e}$ be a tetrahedral element with a face on the curved boundary, and $\boldsymbol{S}$ its NURBS parametrization. The curved tetrahedral face is defined as the image of a straight-sided triangle $\Lambda_{e}$ (in the parametric space of the NURBS) by the NURBS parametrization $S$. Then, the following mapping is considered

$$
\begin{aligned}
\boldsymbol{\theta}: \Lambda_{e} \times[0,1] & \longrightarrow \Omega_{e} \\
(\lambda, \kappa, \vartheta) & \longmapsto \boldsymbol{\theta}(\lambda, \kappa, \vartheta):=(1-\vartheta) \boldsymbol{S}(\lambda, \kappa)+\vartheta \boldsymbol{x}_{4},
\end{aligned}
$$




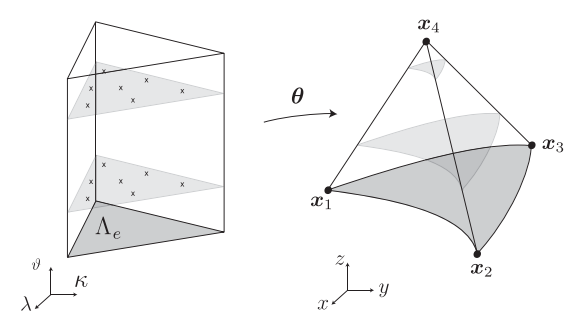

Figure 8. Transformation from $\Lambda_{e} \times[0,1]$ to $\Omega_{e}$ for numerical integration in the 3D NEFEM.

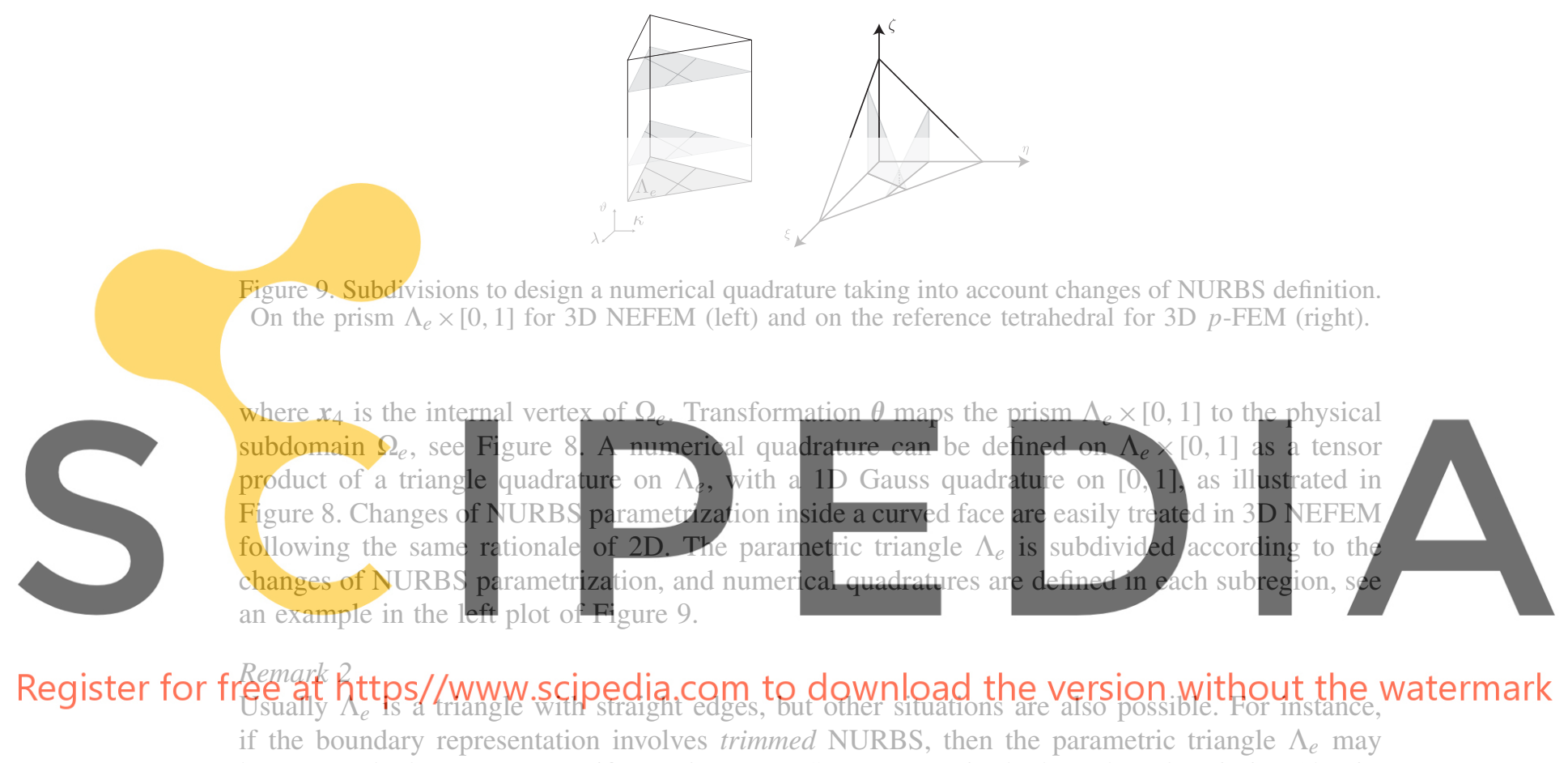

have curved edges. Moreover, if singular NURBS are present in the boundary description (that is, a NURBS containing a point where a directional derivative in the parametric space is zero, and therefore knot lines converge toward a so-called singular point), $\Lambda_{e}$ may be a quadrilateral in the parametric space of the NURBS. In such situations, the mapping of Equation (8) is also valid, and it is only necessary to modify the quadrature of $\Lambda_{e}$, see [22].

In the 3D $p$-FEM context, the definition of a numerical quadrature on the reference tetrahedral accounting changes of NURBS parametrization is more complicated. The generalization of the 2D strategy requires subdivision of the reference tetrahedral element to account for changes of NURBS surface parametrization, see an example in the right plot of Figure 9. In general, different subregions are possible after subdivision. Thus, a simple option to define a quadrature on the reference element is to use further subdivision to obtain only tetrahedral subregions. Then, a composite quadrature may be defined on the reference element based on standard tetrahedral quadratures. In fact, a usual practice to facilitate the implementation of the 3D $p$-FEM is to consider a polynomial approximation of the boundary. For instance, in [25] a least-squares approximation of the exact boundary is considered in a $p$-FEM context. Although the polynomial approximation of the boundary can be selected to satisfy continuity requirements across element interfaces [26], the exact boundary representation is lost in order to simplify the computational implementation.

To conclude, the design of a numerical quadrature accounting for changes of NURBS definition requires specific strategies. For NEFEM, the complexity of the numerical integration in 3D domains is reduced to the design of a $2 \mathrm{D}$ numerical quadrature on the parametric triangle $\Lambda_{e}$, and exact 
Table I. Comparison of FE techniques used in domains with curved boundaries.

\begin{tabular}{lcc}
\hline & Exact geometry & Consistency \\
\hline Isoparametric FEM & NO & NO \\
Cartesian FEM & NO & YES \\
$p$-FEM & YES & NO \\
NEFEM & YES & YES \\
\hline
\end{tabular}

integration is feasible in the third direction, see Figures 8 and 9. In contrast, the design of a numerical quadrature in the 3D $p$-FEM requires careful attention. The reference element must be partitioned and 3D quadratures must be considered to define a suitable quadrature in the reference element accounting for changes of NURBS definition, see right plot in Figure 9.

\subsection{Critical comparison}

The main differences between all the FE techniques considered in this work are summarized in Table I. On one hand, the use of a non-linear mapping relating local and Cartesian coordinates (mapping of Equation (2) in the isoparametric FEM and the exact mapping of Equation (6) in the $p$-FEM) induces a loss of consistency. That is, a polynomial interpolation of degree $p>1$ in local coordinates, $\boldsymbol{\xi}$, does not correspond to a polynomial interpolation of degree $p$ in Cartesian coordinates $x$. On the other hand, the use of the isoparametric mapping to perform the numerical integration (as done in the isoparametric FEM and in the Cartesian FEM) introduces geometric errors, i.e. the boundary of the computational domain, $\partial \Omega_{h}$, is a piecewise polynomial

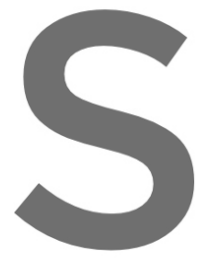
approximation of approximation (for Table I.

It is worth mentioning that, from a computation basis in local coordinates $\xi$, as clone in the isoparan

extra efficiency. In this case the polynomial basis is defined once in the reference element and used to define the approximation in each curved element, whereas a Cartesian approximation requires a

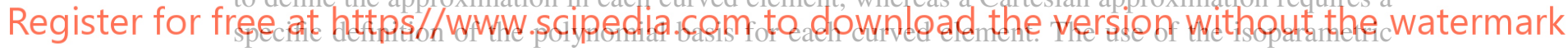
transformation to perform the numerical integration, as done in the isoparametric FEM and in the Cartesian FEM, also induces another marginal extra efficiency. A numerical quadrature is defined in the reference element $I$ and used for each curved element. Whereas methods with an exact boundary representation require specific strategies for curved elements. Nevertheless, it is important to recall that this extra cost is restricted to elements affected by the NURBS boundary representation, in most applications a very small portion of the total number of elements, those in contact with non polynomial boundaries.

A priori error estimates for the FE methodologies considered in this work have similar expressions, with optimal convergence in all cases. However, the hypotheses to obtain these estimates are different, depending on the definition of the approximation, in local or Cartesian coordinates, and on the boundary representation, that is, approximated or exact.

The influence of the definition of the polynomial basis on local or Cartesian coordinates is discussed first. When the polynomial basis is defined with local coordinates $\xi$, the mapping relating local and Cartesian coordinates must be smooth enough to guarantee optimal convergence. In practice, for the isoparametric FEM specific nodal distributions on curved elements are necessary to obtain optimal convergence rates with $p>2$, see Remark 1 and [20,21]. For $p$-FEM, the NURBS parametrization of the curved boundary must be smooth enough to guarantee the necessary smoothness of the $p$-FEM mapping relating local and Cartesian coordinates, see [27]. In contrast, when the polynomial basis is defined with Cartesian coordinates $\boldsymbol{x}$, the derivation of a priori error estimates is very close to FE a priori error estimates in polygonal domains, which can be found in $[28,29]$. For Cartesian FEM and NEFEM, no specific nodal distributions in curved elements are necessary to achieve optimal convergence. Moreover, smooth variations of the NURBS 
parametrization are not required to obtain the optimal convergence rates with NEFEM, see [3]. Nevertheless, optimal a priori error estimates for FE methods with a Cartesian approximation require extra attention if a standard (continuous) Galerkin formulation is considered. If a strong imposition of Dirichlet boundary conditions is considered, or if curved internal edges/faces are present in the mesh, optimal nodal distributions in curved elements are necessary in order to guarantee optimal rates of convergence, see $[3,15]$ and references therein. The key issue is that test functions do not vanish over the curved boundary, even if the test function is associated with a node that is not located on the boundary. With specific nodal distributions, such as Fekette points, the error induced by this lack of consistency is lower than the approximation error and the optimal convergence is guaranteed. Obviously, this is not the case for weakly imposed Dirichlet boundary conditions, where optimal convergence is obtained irrespective of the node placement. Recall that in a DG framework boundary conditions are usually imposed in a weak sense, and recent studies also suggest advantages of imposing boundary conditions weakly in a standard continuous Galerkin framework, see [30].

The influence of the boundary representation on the convergence properties of the approximation

is discussed next. For FE methods with an approximate boundary representation (isoparametric FEM and Cartesian FEM), optimal convergence is provided under the assumption that geometric errors are lower than the discretization error. The difference between the computational element $\Omega_{e}^{h}$ and the physical subdomain $\Omega_{e}$ must be bounded by $\gamma h^{p}$, where $\gamma$ is a constant, $h$ is the characteristic mesh size, and $p$ is the interpolation degree. Moreover, bounds of the Jacobian of the isoparametric transformation and its first $p$ derivatives are also necessary, see [20].

Thus, a curved element with an approximated boundary representation must verify two contradictory requirements. On one hand, the computational (polynomial) boundary $\partial \Omega^{h}$ has to be close
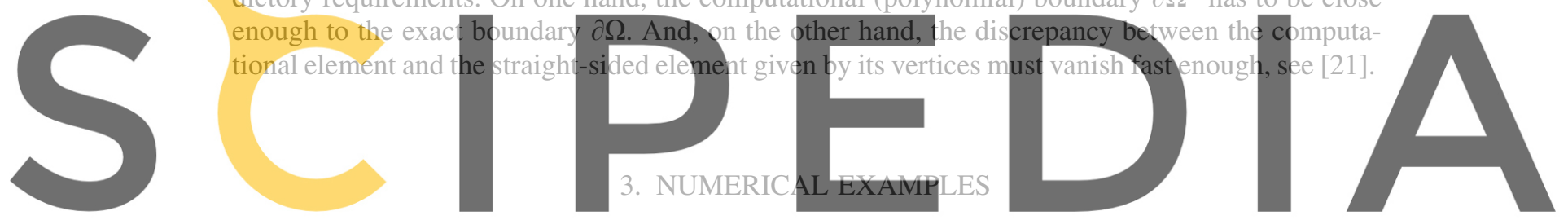

Two numerical examples are considered in order to compare the FE methodologies discussed in

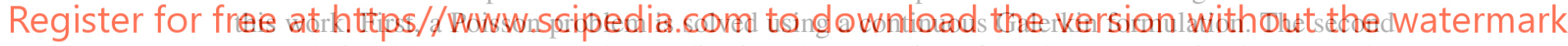
example involves a more complex application, the scattering of an electromagnetic plane wave by a perfectly conducting obstacle, which is solved using a DG formulation.

\subsection{Poisson problem}

The following model problem is considered:

$$
\begin{aligned}
-\Delta u=f & \text { in } \Omega, \\
u=u_{d} & \text { on } \Gamma_{d}, \\
\nabla u \cdot \boldsymbol{n}=g_{n} & \text { on } \Gamma_{n},
\end{aligned}
$$

where $\Omega$ is the domain (see two computational meshes with curved FEs in Figure 10), $\bar{\Gamma}_{d} \cup \bar{\Gamma}_{n}=\partial \Omega$ and $\boldsymbol{n}$ is the outward unit normal vector on $\partial \Omega$. A Dirichlet boundary condition, corresponding to the analytical solution, is imposed in strong form on the polygonal part of the boundary $\Gamma_{d}$, and a Neumann boundary condition, also corresponding to the analytical normal flux, is imposed on the curved part of the boundary $\Gamma_{n}$. If desired, Dirichlet boundary conditions could be imposed in a strong sense over the curved boundary by considering Fekette nodal distributions on curved boundary edges, see Section 2.4. The curved part of the boundary is given by the usual quadratic NURBS that describes a circle, see [6], trimmed to represent half a circle.

A polynomial source term is considered first, $f(x, y)=-\left(32 x^{3} y^{2}+6 x y^{4}+2 x^{5}+42 y^{5}\right)$, in order to illustrate both the relevance of an accurate boundary representation of the domain and the issue 

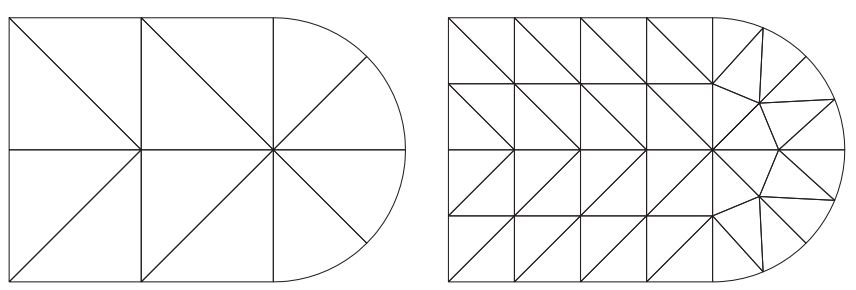

Figure 10. Coarse meshes for the Poisson problem. Nested remeshing is used for refinement.

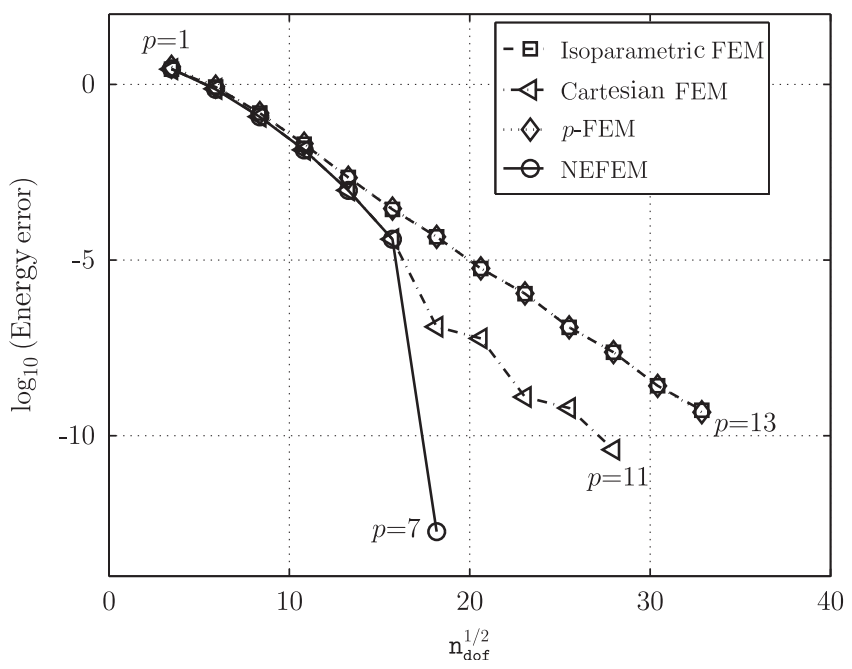

Figure 11. Poisson problem with polynomial analytical solution: $p$-convergence in the energy norm in the coarse mesh of Figure 10.

of consistency. The analytical solution of the problem is a polynomial function of degree 7 , namely

$$
u(x, y)=x^{5} y^{2}+x^{3} y^{4}+y^{7} .
$$

Figure 11 shows a $p$-convergence comparison in the coarse mesh of Figure 10. The energy error is represented as a function of the square root of the number of degrees of freedom ( $\left.n_{\text {dof }}\right)$ when the polynomial order of the approximation is uniformly increased, starting with $p=1$. In NEFEM, the boundary of the domain is exactly represented and the polynomial basis for the approximation is defined in Cartesian coordinates. Therefore, with a polynomial approximation of degree $p=7$ the solution provided by NEFEM is the exact solution (except from rounding errors). With Cartesian FEs, the basis is also defined in Cartesian coordinates, but the computational boundary is a piecewise polynomial approximation of the circle. Thus, the difference between NEFEM and Cartesian FEM is only due to geometric errors. Although isoparametric FE and the $p$-FEM show the expected (exponential) convergence, the effect of a non-consistent approximation is clearly observed. The function to be approximated is a polynomial in Cartesian coordinates $u(\boldsymbol{x})$, but it is far from being a polynomial function in local coordinates $u(\xi(x))$. In this example, errors introduced by a non-consistent approximation are higher than geometric errors. Thus, isoparametric FEM and $p$-FEM provide the same performance.

Next, the same Poisson problem is solved with a non-polynomial source term, $f(x, y)=$ $x \cos (y)+y \sin (x)$, such that the analytical solution of the problem is

$$
u(x, y)=x \cos (y)+y \sin (x) .
$$

Convergence under $h$-refinement is first explored. Figure 10 shows the first two computational meshes; nested remeshing is used for refinement. The number of integration points is sufficiently large in order to ensure that no errors due to numerical integration are present. Energy error is 

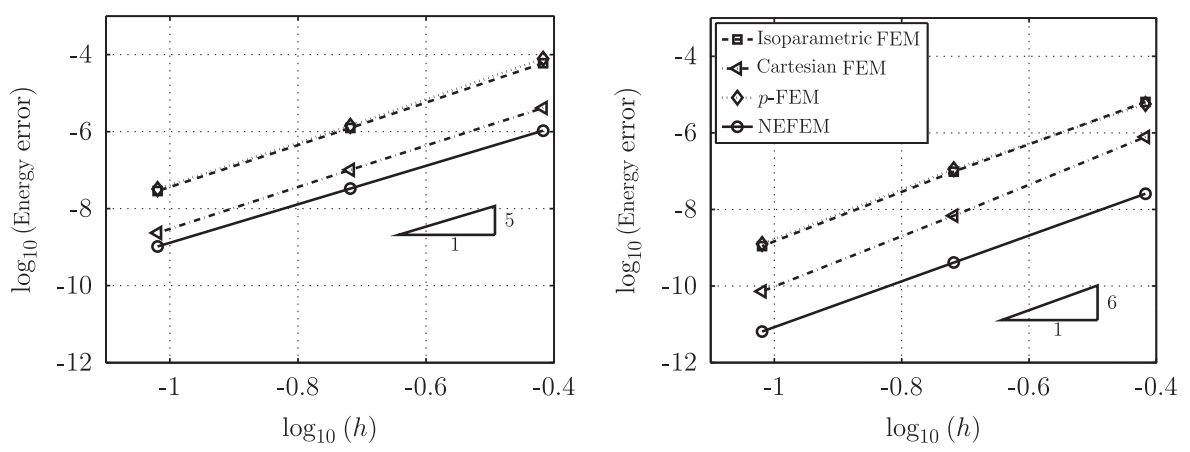

Figure 12. Poisson problem: $h$-convergence in the energy norm for $p=5$ (left) and $p=6$ (right).
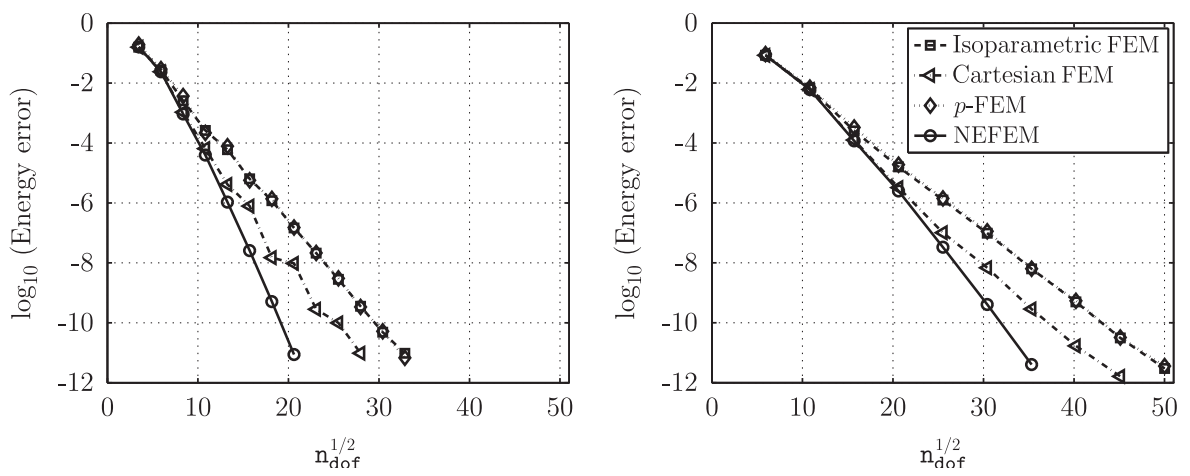

Figure 13. Poisson problem: $p$-convergence in the energy norm for the computational meshes represented in Figure 10.

depicted in Figure 12 for a polynomial approximation of degree $p=5$ and $p=6$. The optimal rate of $h$-convergence is exhibited by every FE technique considered, but some differences in accuracy are observed. In this example the use of a Cartesian approximation (Cartesian FEs and NEFEM) provides more accurate results than defining the approximation with local coordinates. NEFEM always provides the most accurate results due to the combined effect of the Cartesian approximation and exact boundary representation. With $p=6$, NEFEM is one order of magnitude more accurate than Cartesian FEs and two orders of magnitude more accurate than isoparametric FEs and $p$-FEM. In this example, $p$-FEM does not represent an advantage with respect to isoparametric FEs. The error induced by the geometric approximation of the boundary is lower than the error introduced by the definition of the polynomial basis in local coordinates.

As shown in the $h$-convergence study, NEFEM is advantageous for high-order approximations. Next, convergence under $p$-refinement is explored and compared. Figure 13 represents the evolution of the energy error as a function of the square root of the $n_{\text {dof }}$. The polynomial degree of the approximation is uniformly increased starting with $p=1$ and for the discretizations shown in Figure 10. As the order of the polynomial approximation is increased, NEFEM offers the best performance. In fact, the desired error is attained with the minimum $\mathrm{n}_{\text {dof }}$. Figure 13 shows that, for a given accuracy and the coarsest mesh in Figure 10, NEFEM allows to reduce drastically the $\mathrm{n}_{\text {dof }}$. In particular, a reduction of $40 \%$ compared to Cartesian FEM and up to $50 \%$ compared to isoparametric FEM or $p$-FEM.

Finally, the influence of the number of integration points $n_{i p}$ on the accuracy is studied. The coarsest mesh in Figure 10 with a polynomial approximation of degree $p=6$ and $p=8$ is used. To study quadrature accuracy, Figure 14 shows the evolution of the energy error versus the number of Gauss integration points for every curved boundary edge. When the polynomial basis is defined in Cartesian coordinates (Cartesian FEM and NEFEM), numerical integration requires more integration points to reach its maximum accuracy, compared to the other methods. For a 

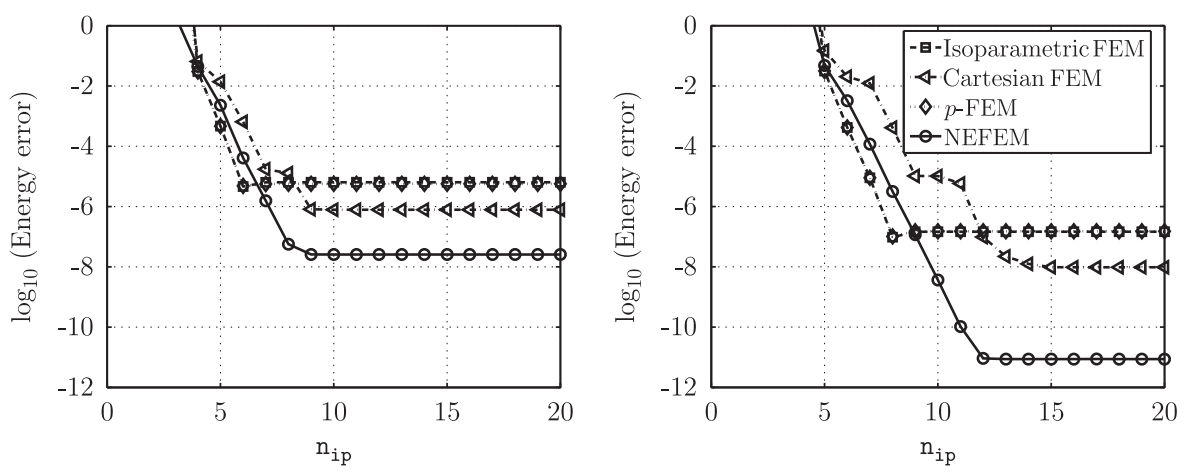

Figure 14. Poisson problem: energy norm of the error as the number of integration points $\left(n_{i p}\right)$ along the curved boundary edge is increased. Coarse mesh of Figure 10 with $p=6$ (left) and $p=8$ (right).

given degree of interpolation, NEFEM is able to reach the same accuracy of isoparametric FEs with only one extra integration point. Moreover, with three or four integration points more than isoparametric FEM, NEFEM reaches its maximum accuracy. For a degree of interpolation $p=8$, NEFEM is four orders of magnitude more precise than isoparametric FEM and $p$-FEM, and three orders of magnitude more precise than Cartesian FEM.

Comparing left and right plots in Figure 14 it is important to note that NEFEM with $p=6$ and 9 Gauss integration points along curved boundary edges achieves comparable accuracy to isoparametric FEM with $p=8$ and 9 Gauss integration points. This means that NEFEM is able to reach the same accuracy than isoparametric FEs using the same number of integration points for boundary integrals but with a lower degree of approximation. Figure 15 shows the evolution of the energy error versus the total number of integration points for interior integrals as the degree of approximation $p$ is increased. For each point of this figure, the minimum number of integration points to achieve maximum accuracy for a given $p$ is used. Owing to the lower degree of approximation required by NEFEM, it achieves the same accuracy than isoparametric FEM with an important reduction in the total number of integration points. Therefore, although NEFEM requires more computational effort per integration point due to the Cartesian approximation and the NURBS boundary representation, this comparison shows that NEFEM is competitive because the required number of integration points is substantially reduced to achieve a desired accuracy. Figure 15 also shows that Cartesian FEs are not competitive because the necessary number of integration points to achieve a comparable accuracy is much greater than using NEFEM or isoparametric FEM. Finally, note that the results using $p$-FEM are not displayed because in this problem it behaves as isoparametric FEM, see Figure 14.

\subsection{Electromagnetic scattering problem}

The second example considers the scattering of an electromagnetic plane wave, traveling in the $x^{+}$ direction, by a perfect electric conducting circular cylinder. The wavelength of the incident field is denoted by $\lambda$ and the diameter of the circle is $4 \lambda$. The problem is solved in the time domain using a DG formulation, see [31,32]. A coarse mesh with only four elements for the discretization of the curved boundary is considered, and high-order approximations are used to properly capture the solution. Figure 16 shows the computational mesh, the transverse scattered field computed with NEFEM and $p=10$, and the radar cross section (RCS, see [33]). A perfectly matched layer (PML) is introduced in order to absorb outgoing waves. Note that a $2 \lambda$ thick PML is considered, which is enough to ensure that the accuracy comparison is not affected by the PML.

The RCS error evolution for increasing $p$ is depicted in Figure 17. For the same discretization (i.e. same degree of interpolation), NEFEM results are more accurate than isoparametric or Cartesian FEs, with an approximate boundary description, and also more accurate than $p$-FEM, with an exact boundary representation. For instance, NEFEM with $p=10$ produces a RCS error in $\mathscr{L}^{2}([-\pi, \pi])$ norm of about $10^{-2}$, whereas isoparametric or Cartesian FEs require $p=12$ to achieve a comparable accuracy, and $p$-FEM requires $p=11$. Thus, NEFEM is able to reach the desired accuracy with a 


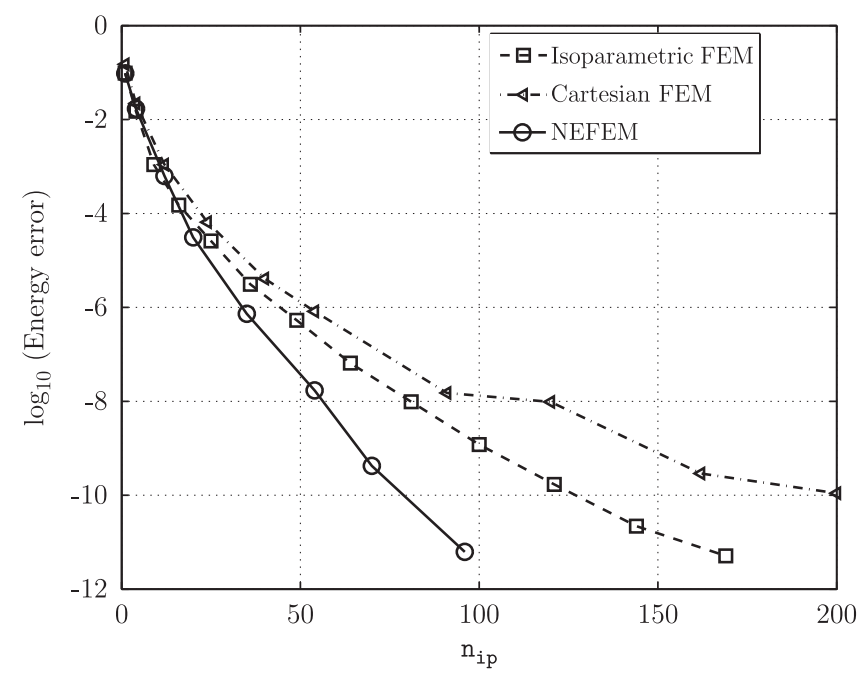

Figure 15. Poisson problem: energy norm of the error versus number of integration points $\left(\mathrm{n}_{\mathrm{ip}}\right)$ for interior integrals as $p$ increases using the coarse mesh of Figure 10.

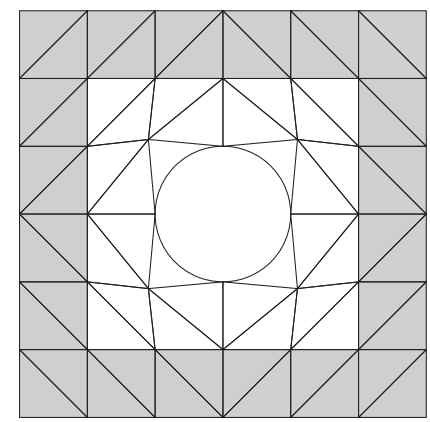

(a)

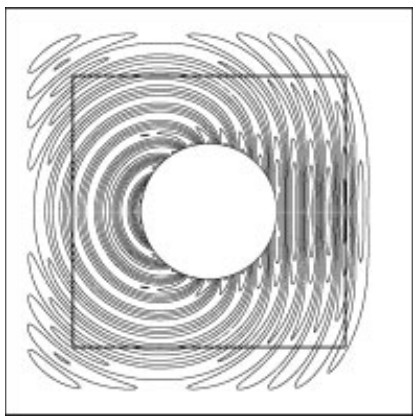

(b)

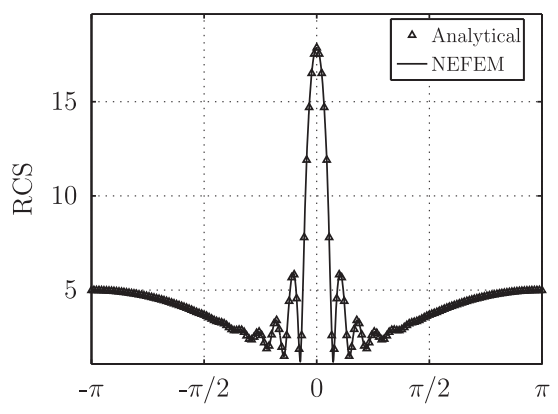

(c)

Angle

Figure 16. Scattering by a perfect conductor circle: (a) computational mesh with a $2 \lambda$ thick PML; (b) transverse scattered field for a NEFEM solution with $p=10$; and (c) RCS.

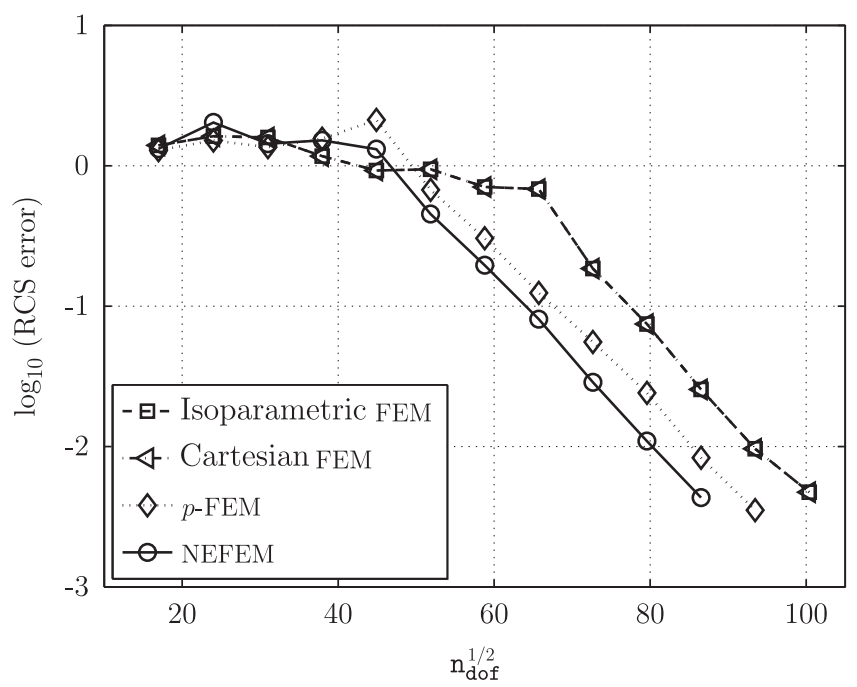

Figure 17. Scattering by a perfect conductor circle: RCS error in $\mathscr{L}^{2}(-\pi, \pi)$ norm as the polynomial order of the approximation is increased. 
reduction in $\mathrm{n}_{\text {dof }}$ of about $30 \%$ compared to isoparametric or Cartesian FEs, and of $15 \%$ compared to $p$-FEM (also with an exact boundary representation). This difference in number of degrees of freedom implies important differences in computational cost. NEFEM computation requires 2585 time steps to reach the time-harmonic steady state, whereas isoparametric and Cartesian FEs employ 3692 time steps and $p$-FEM requires 3114 time steps. In addition, each time step with NEFEM requires less computational cost due to the lower $p$ needed to achieve the desired accuracy.

The difference between isoparametric FEs and Cartesian FEs is indistinguishable, showing that a Cartesian approximation of the solution does not offer any advantage if an approximated boundary representation is considered. The difference between isoparametric FEs and $p$-FEM is only due to geometric errors, and relevant differences in accuracy are observed. Finally, NEFEM also considers the exact boundary representation as $p$-FEM and outperforms it. The Cartesian approximation combined with an exact boundary representation (i.e. NEFEM) provides in this example the maximum accuracy for a given spatial discretization. Finally, note that with an approximate boundary representation the exponential convergence is exhibited for $p>8$, whereas with an exact boundary representation the exponential convergence is achieved for $p>5$.

To conclude, it is worth remarking that only one element per two wavelengths is considered in this example and a RCS error below $10^{-2}$ is obtained with $p=11$, that is, using 6 nodes per wavelength. Thus, the exact geometry considered in NEFEM combined with the Cartesian approximation allow to compute accurate solutions with the minimum $\mathrm{n}_{\mathrm{dof}}$, compared to other curved FEs and other techniques used by the computational electromagnetics community. For instance, Vinh et al. [34] use 22 nodes per wavelength and low-order finite differences, El Hachemi et al. [35] use 20 nodes per wavelength and linear FEs, and Cioni et al. [36] use 20 nodes per wavelength and finite volumes.

\section{CONCLUDING REMARKS}

This paper presents a comparison between several FE methodologies for the treatment of curved boundaries: classical isoparametric FEM and Cartesian FEM with an approximated boundary representation, and $p$-FEM and NEFEM with an exact description of the boundary. A critical comparison of these methods is presented, with particular emphasis on two issues: consistency of the approximation and exact geometric description. Special attention is paid to the numerical integration for FE methods with an exact boundary representation with NURBS. In this case, numerical integration must be designed to take into account the piecewise nature of the NURBS parametrization. The strategy to perform the numerical integration in $p$-FEM and NEFEM is discussed for both 2D and 3D domains.

2D numerical examples are presented, using continuous and DG formulations, in order to investigate the performance and benefits of each methodology. The main conclusion of this study is that NEFEM provides maximum accuracy for a given spatial discretization, showing the importance of a consistent approximation and exact boundary representation. For a Poisson problem, NEFEM provides results at least two orders of magnitude more accurate than the other FE methods studied here. Ensuring consistency is an important issue in this example, and Cartesian FEM performs better than classical isoparametric FEM or $p$-FEM. The second example considers an electromagnetic scattering application in which the quantity of interest is defined along the curved boundary. In this case, the use of an exact representation of the geometry is crucial. Again, NEFEM provides the most accurate results for a given spatial discretization, one order of magnitude more precise than classical isoparametric FEM or Cartesian FEM, and also more accurate than methods considering an exact boundary representation, such as $p$-FEM.

\section{REFERENCES}

1. Szabó B, Babuška I. Finite Element Analysis. Wiley: New York, 1991.

2. Xue D, Demkowicz L. Control of geometry induced error in hp finite element (FE) simulations. I. Evaluation of FE error for curvilinear geometries. International Journal for Numerical Analysis and Modelling 2005; 2(3):283-300. 
3. Sevilla R, Fernández-Méndez S, Huerta A. NURBS-enhanced finite element method (NEFEM). International Journal for Numerical Methods in Engineering 2008; 76(1):56-83.

4. Szabó B, Düster, Rank E. The p-version of the finite element method. (Fundamentals) of Encyclopedia of Computational Mechanics, Chapter 5, vol. 1. Wiley: New York, 2004.

5. Gordon WJ, Hall CA. Transfinite element methods: blending-function interpolation over arbitrary curved element domains. Numerische Mathematik 1973; 21:109-129.

6. Piegl L, Tiller W. The NURBS Book. Springer: London, 1995.

7. Jörg P, Ulrich R. Subdivision surfaces. Geometry and Computing, vol. 3. Springer: Berlin, 2008.

8. Kagan P, Fischer A, Bar-Yoseph PZ. New B-spline finite element approach for geometrical design and mechanical analysis. International Journal for Numerical Methods in Engineering 1998; 41:435-458.

9. Cirak F, Ortiz M, Schröder P. Subdivision surfaces: a new paradigm for thin-shell finite-element analysis. International Journal for Numerical Methods in Engineering 2000; 47(12):2039-2072.

10. Inoue K, Kikuchi Y, Masuyama T. A NURBS finite element method for product shape design. Journal of Engineering Design 2005; 16(2):157-174.

11. Hughes TJR, Cottrell JA, Bazilevs Y. Isogeometric analysis: CAD, finite elements, NURBS, exact geometry and mesh refinement. Computer Methods in Applied Mechanics and Engineering 2005; 194(39-41):4135-4195.

12. Schramm U, Pilkey W. The coupling of geometric descriptions and finite elements using NURBS: a study of shape optimization. Finite Elements in Analysis and Design 1993; 15(1):11-34.

13. Sevilla R, Fernández-Méndez S, Huerta A. NURBS-enhanced finite element method (NEFEM) for Euler equations. International Journal for Numerical Methods in Fluids 2008; 57(9):1051-1069.

14. Kima H-J, Seoa Y-D, Youn S-K. Isogeometric analysis for trimmed CAD surfaces. Computer Methods in Applied Mechanics and Engineering 2009; 198(37-40):2982-2995.

15. Sevilla R. NURBS-enhanced finite element method (NEFEM). Ph.D. Thesis, Universitat Politènica de Catalunya, 2009.

16. Ergatoudis J, Irons BM, Zienkiewicz OC. Curved isoparametric ‘quadrilateral' elements for finite element analysis. International Journal of Solids and Structures 1968; 4(1):31-42.

17. Zienkiewicz OC. The Finite Element Method in Engineering Science (2nd edn). McGraw-Hill: London, 1971.

18. Wandzura S, Xiao H. Symmetric quadrature rules on a triangle. Computers and Mathematics with Applications 2003; 45(12):1829-1840.

19. Zienkiewicz OC, Taylor RL. The basis. The Finite Element Method (5th edn), vol. 1. Butterworth-Heinemann: London, 2000.

20. Ciarlet PG, Raviart PA. Interpolation theory over curved elements, with applications to finite element methods. Computer Methods in Applied Mechanics and Engineering 1972; 1(1):217-249.

21. Lenoir M. Optimal isoparametric finite elements and error estimates for domains involving curved boundaries. SIAM Journal on Numerical Analysis 1986; 23(3):562-580.

22. Sevilla R, Fernández-Méndez S, Huerta A. 3D NURBS-enhanced finite element method (NEFEM). International Journal for Numerical Methods in Engineering, DOI: 10.1002/nme.3164.

23. Pascal JF, George P-L. Maillages: Applications aux éléments Finis. Hermès Science Publications: Paris, 1999.

24. Banerjee U, Suri M. The effect of numerical quadrature in the $p$-version of the finite element method. Mathematics of Computation 1992; 59(199):1-20.

25. Coyle J, Ledger P. Evidence of exponential convergence in the computation of Maxwell eigenvalues. Computer Methods in Applied Mechanics and Engineering 2005; 194(2-5):587-604.

26. Luo X-J, Shephard MS, Remacle J-F. Influence of geometric approximation on the accuracy of higher order methods. Technical Report 1, SCOREC, 2001.

27. Babuška I, Suri M. The optimal convergence rate of the $p$-version of the finite element method. SIAM Journal on Numerical Analysis 1987; 24(4):750-776.

28. Johnson C. Numerical Solution of Partial Differential Equations by the Finite Element Method. Cambridge University Press: Cambridge, 1987.

29. Brenner SC, Scott LR. The Mathematical Theory of Finite Element Methods. Springer: Berlin, 1994.

30. Bazilevs Y, Hughes TJR. Weak imposition of Dirichlet boundary conditions in fluid mechanics. Computers and Fluids 2007; 36(1):12-26.

31. Cockburn B. Discontinuous Galerkin methods for computational fluid dynamics. In Fluids, Encyclopedia of Computational Mechanics, Stein E, de Borst R, Hughes TJR (eds), Chapter 4, vol. 3. Wiley: New York, 2004.

32. Hesthaven JS, Warburton T. Nodal Discontinuous Galerkin Methods: Algorithms, Analysis, and Applications. Texts in Applied Mathematics, vol. 54. Springer: Berlin, 2008.

33. Balanis CA. Advanced Engineering Electromagnetics. Wiley: New York, 1989.

34. Vinh H, van Dam CP, Dwyer HA. Finite difference Maxwell solver to study geometric shape effects on radar signature. Journal of Aircraft 1997; 34(1):56-63.

35. El Hachemi M, Hassan O, Morgan K, Rowse D, Weatherill N. A low-order unstructured-mesh approach for computational electromagnetics in the time domain. Philosophical Transactions of the Royal Society of London Series A: Mathematical, Physical and Engineering Science 2004; 362(1816):445-469.

36. Cioni JP, Fezoui L, Steve H. A parallel time-domain Maxwell solver using upwind schemes and triangular meshes. IMPACT of Computing in Science and Engineering 1993; 5(3):215-247. 